\title{
Monounireducible Nonhomogeneous Continuous Time Semi-Markov Processes Applied to Rating Migration Models
}

\author{
Guglielmo D'Amico, ${ }^{1}$ Jacques Janssen, $^{2}$ and Raimondo Manca ${ }^{3}$ \\ ${ }^{1}$ Department of Pharmacy, University "G. d'Annunzio" of Chieti, Via dei Vestini 31, 66013 Chieti, Italy \\ ${ }^{2}$ CESIAF, EURIA, University of Bretagne Occidentale, 6 Avenue L. Gorgeu, CS 93837, \\ 29238 Brest Cedex 3, France \\ ${ }^{3}$ MEMOTEF Department, University "La Sapienza" of Roma, Via del Castro Laurenziano 9, \\ 00161 Roma, Italy
}

Correspondence should be addressed to Guglielmo D’Amico, g.damico@unich.it

Received 3 April 2012; Accepted 11 September 2012

Academic Editor: C. D. Lai

Copyright (c) 2012 Guglielmo D'Amico et al. This is an open access article distributed under the Creative Commons Attribution License, which permits unrestricted use, distribution, and reproduction in any medium, provided the original work is properly cited.

Monounireducible nonhomogeneous semi- Markov processes are defined and investigated. The mono- unireducible topological structure is a sufficient condition that guarantees the absorption of the semi-Markov process in a state of the process. This situation is of fundamental importance in the modelling of credit rating migrations because permits the derivation of the distribution function of the time of default. An application in credit rating modelling is given in order to illustrate the results.

\section{Introduction}

Semi-Markov processes (SMPs) are a generalization of Markov processes in which the waiting time distributions before the occurrence of a transition are modelled by any kind of distribution function; see [15]. This means that, on the contrary of Markov processes, it is possible to use also no memoryless distributions which determine a duration effect.

The duration effect affirms that the time the system is in a state influences the system's transition probability. One way to detect and quantify this effect, in a SMP, is by using backward and forward recurrence time processes associated to the SMP.

In $[5,10]$ general distributions of the transition probabilities of SMP with backward and forward times are investigated for discrete time nonhomogeneous and for continuous time homogeneous processes, respectively. In these papers a credit risk application is also 
described. In this paper a further generalization is presented. In fact, a duration-dependent semi-Markov model is presented for continuous time nonhomogeneous processes. This extension is motivated by theoretical reasons as well by the practical need of making an efficient rating migration model available. In the paper the mono-unreducible topological structure for nonhomogeneous continuous time semi-Markov processes (NHCTSMPs) is introduced and analysed. It represents a sufficient condition for the absorption of the process in the down states of the system. Then, it represents the ideal environment in which to treat credit risk models.

The paper is organized as follows. Section 2 gives definitions and notations on NHCTSMP and introduces backward and forward processes and their joint distributions together with that of the SMP. Section 3 studies the monounreducible topological structure and derives the distribution function of going into default state. Section 4 presents a credit risk model and a numerical example.

\section{Continuous Time Non-Homogeneous Semi-Markov Process}

Let us consider two sequences of random variables defined on a complete; filtered probability space $\left(\Omega, \mathcal{F}, \mathcal{F}_{t}, P\right)$ as the following:

(i) $J_{n}: \Omega \rightarrow I=\{1,2, \ldots, m\}, n \in \mathrm{N}$ representing the state at the $n$th transition;

(ii) $T_{n}: \Omega \rightarrow \mathbf{R}^{+}$representing the time of the $n$th transition.

We suppose that $\left(J_{n}, T_{n}\right)$ is a Markov renewal process of kernel $\mathbf{Q}=\left[Q_{i j}(s, t)\right]$ :

$$
\begin{array}{r}
P\left[J_{n+1}=j, T_{n+1} \leq t \mid \sigma\left(J_{a}, T_{a}\right), J_{n}=i, T_{n}=s, 0 \leq a \leq n\right] \\
=P\left\{J_{n+1}=j, T_{n+1} \leq t \mid J_{n}=i, T_{n}=s\right\}:=Q_{i j}(s, t) .
\end{array}
$$

We also know that.

$$
p_{i j}(s):=P\left\{J_{n+1}=j \mid J_{n}=i, T_{n}=s\right\}=\lim _{t \rightarrow \infty} Q_{i j}(s, t) .
$$

$\mathbf{P}(s)=\left[p_{i j}(s)\right]$ is the transition matrix of the embedded Markov chain $\left\{J_{n}\right\}$.

Let us introduce the probability; the process will leave state $i$ within time $t$ :

$$
H_{i}(s, t):=P\left\{T_{n+1} \leq t \mid J_{n}=i, T_{n}=s\right\}=\sum_{k \in I} Q_{i k}(s, t)
$$

It is possible to define the distribution function of the waiting time in each state $i$, given that the state, successively, occupied is known

$$
G_{i j}(s, t):=P\left\{T_{n+1} \leq t \mid J_{n}=i, J_{n+1}=j, T_{n}=s\right\} .
$$


The related probabilities can be obtained by means of the following formula:

$$
G_{i j}(s, t)= \begin{cases}\frac{Q_{i j}(s, t)}{p_{i j}(s)}, & \text { if } p_{i j}(s) \neq 0, \\ 1, & \text { if } p_{i j}(s)=0 .\end{cases}
$$

Denote by $N(t)=\sup \left\{n: T_{n} \leq t\right\}$, the NHCTSMP $Z=\left(Z(t), t \in \mathbf{R}^{+}\right)$is defined as $Z(t)=J_{N(t)}$. It represents for each waiting time the state occupied by the embedded Markov chain.

The transition probabilities of $Z(t)$ are defined in the following way:

$$
\phi_{i j}(s, t):=P\left\{Z(t)=j \mid Z(s)=i, T_{N(s)}=s\right\} .
$$

They are obtained by solving the following system of integral equations:

$$
\phi_{i j}(s, t)=\delta_{i j}\left(1-H_{i}(s, t)\right)+\sum_{k \in I} \int_{s}^{t} \dot{Q}_{i k}(s, \theta) \phi_{k j}(\theta, t) d \theta,
$$

where $\delta_{i j}$ represents the Kronecker symbol.

The part $\delta_{i j}\left(1-H_{i}(s, t)\right)$ of formula (2.7) gives the probability that the process does not have transitions up to time $t$ given that it starts in state $i$ at time $s$.

The term $\sum_{k \in I} \int_{s}^{t} \dot{Q}_{i k}(s, \theta) \phi_{k j}(\theta, t) d \theta$ considers the permanence of the system in state $i$ up to the time $\theta$, where a transition in state $k$ occurs. After the transition, the system will move to state $j$ following one of all the possible trajectories going from state $k$ at time $\theta$ to state $j$ at time $t$. All possible states $k$ and times $\theta$ are considered by the summation and the integration.

With the aim of considering a general duration-dependent model, which is of fundamental importance in many applicative domains such as credit rating modelling and reliability theory, we introduce the recurrence time processes. These processes were investigated by many authors; see, for example, [4-6].

Given $\left(J_{n}, T_{n}\right)$, we define the following recurrence processes:

$$
B(t)=t-T_{N(t)}, \quad F(t)=T_{N(t)+1}-t .
$$

$B(t)$ is called the non-homogeneous backward time (or age) process, and $F(t)$ is the non-homogeneous forward time (or residual time) process.

The recurrence time processes complement $Z(t)$ to a Markov process with respect to $\mathcal{F}_{t}^{+} \equiv \sigma\{Z(s), F(s), s \in[0, t]\}, t \geq 0$, then for any bounded $I \times \mathbf{R}^{+}$-measurable function $f(x, t)$ it results

$$
E\left[f(Z(t), F(t)) \mid \mathscr{F}_{s}^{+}\right]=E[f(Z(t), F(t)) \mid Z(s), F(s)] .
$$

The relation remains true if we interchange $B(t)$ with $F(t)$; see [7].

Figure 1 represents a trajectory of a SMP and the backward and forward processes. In Figure 1 we have $N(s)=n$ and $N(t)=h$, a starting backward $B(s)=s-T_{n}=s-l$, a starting 


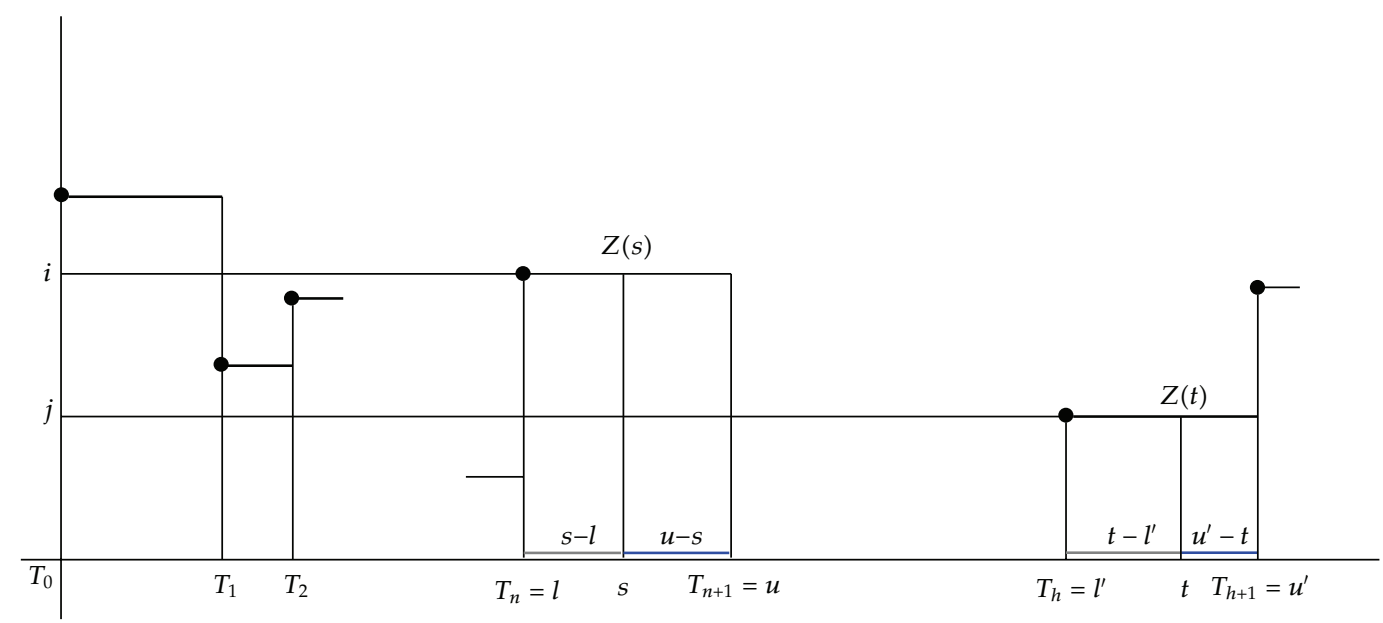

Figure 1: Trajectory of a NHSMP with backward and forward times.

forward $F(s)=T_{n+1}-s=u-s$, a final backward $B(t)=t-T_{h}=t-l^{\prime}$, and a final forward $F(t)=T_{h+1}-t=u^{\prime}-t$.

The transition probabilities of a SMP change in function of the values of the recurrence time processes. In fact the conditional waiting times distribution functions (2.4) can be of any type, and then, also no memoryless distributions can be used. In this case, the time length spent in the starting state (initial backward value) changes the transition probabilities, and the same effect is determined by a starting forward which imposes a constraint on the time of the next transition. The same argument holds for the final backward and forward values.

To understand how the transition probabilities of the SMP are perturbed by imposing constraints on the recurrence processes both at starting and arriving times, we report here some of the results obtained in [8].

Definition 2.1. For all $i, j \in I$ and for all $l \leq s \leq u \leq l^{\prime} \leq t \leq u^{\prime}$ such that $1-H_{i}(l, u)>0$, one defines the transition probabilities with initial and final backward and forward

$$
\begin{aligned}
& { }^{b f} \phi_{i j}^{B F}\left(l, s, u ; l^{\prime}, t, u^{\prime}\right) \\
& \quad=P\left[Z(t)=j, B(t) \leq t-l^{\prime}, F(t) \leq u^{\prime}-t \mid Z(s)=i, B(s)=s-l, F(s)=u-s\right] .
\end{aligned}
$$

The following result holds

Theorem 2.2. For all $i, j \in I$ and for all $l \leq s \leq u \leq l^{\prime} \leq t \leq u^{\prime}$ such that $1-H_{i}(l, u)>0$, one has

$$
\begin{gathered}
{ }^{b f} \phi_{i j}^{B F}\left(l, s, u ; l^{\prime}, t, u^{\prime}\right)=\sum_{k \in I} \frac{d Q_{i k}(l, u)}{d H_{i}(l, u)} \phi_{k j}^{B F}\left(u ; l^{\prime}, t, u^{\prime}\right), \\
\phi_{k j}^{B F}\left(u ; l^{\prime}, t, u^{\prime}\right)=\delta_{k j}\left(H_{k}\left(u, u^{\prime}\right)-H_{k}(u, t)\right) 1_{\left\{l^{\prime}=u\right\}}+\sum_{p \in I} \int_{u}^{t} \dot{Q}_{k p}(u, \theta) \phi_{p j}^{B F}\left(\theta ; l^{\prime}, t, u^{\prime}\right) d \theta .
\end{gathered}
$$

Proof. See [8]. 
Definition 2.3. For all $i, j \in I$ and for all $l \leq s \leq u \leq l^{\prime} \leq t \leq u^{\prime}$ such that $1-H_{i}(l, u)>0$, one defines the probabilities with initial backward-certain forward and final backward-forward

$$
\begin{aligned}
{ }^{b f} \phi_{i j}^{B F} & \left(l, s, u ; l^{\prime}, t, u^{\prime}\right) \\
\quad & =P\left[Z(t)=j, B(t) \leq t-l^{\prime}, F(t) \leq u^{\prime}-t \mid Z(s)=i, B(s)=s-l, F(s)>u-s\right] .
\end{aligned}
$$

Theorem 2.4. For all $i, j \in I$ and for all $l \leq s \leq u \leq l^{\prime} \leq t \leq u^{\prime}$ such that $1-H_{i}(l, u)>0$, one has

$$
{ }^{b F} \phi_{i j}^{B F}\left(l, s, u ; l^{\prime}, t, u^{\prime}\right)=\delta_{i j} \frac{\left(H_{i}\left(l, u^{\prime}\right)-H_{i}(l, t)\right)}{1-H_{i}(l, u)} 1_{\left\{l^{\prime}=l\right\}}+\sum_{m \in I} \int_{u}^{t} \frac{\dot{Q}_{i m}(u, \theta)}{1-H_{i}(l, u)} \phi_{m j}^{B F}\left(\theta ; l^{\prime}, t, u^{\prime}\right) d \theta .
$$

Proof. See [8].

There are several particular cases of (2.14) and (2.11) which can be of interest. In Section 3, some of them will be studied in relation to the credit-rating problem also in the asymptotic case.

\section{Mono-Unireducible Non-Homogeneous Semi-Markov Process}

In this section, we introduce the mono-unireducible topological structure. In [9], it was studied the asymptotic behavior of a mono-unireducible discrete time non-homogeneous SMP. Here, we extend the results to the continuous time case.

Definition 3.1. Let $\mathbf{A}=\left[\begin{array}{cc}\mathbf{A}_{u, u} & \mathbf{A}_{u, D} \\ \mathbf{0}^{T} & A_{D, D}\end{array}\right]$ be a $m \times m$ matrix.

$A$ is mono-unireducible if:

(i) $\mathbf{A}_{U, U}$ is a $(m-1) \times(m-1)$ circuit matrix,

(ii) $\mathbf{A}_{U, D}$ is a $(m-1)$ nonnegative column vector with at least one positive element,

(iii) $A_{D, D}=a_{m m}>0$.

Remark 3.2. The product of a mono-unireducible matrix with a positive diagonal matrix is a mono-unireducible matrix.

Definition 3.3. A NHCTSMP $Z(t)$ is said mono-unireducible if:

(i) for all $s, t \in \mathbf{R}_{+} \dot{Q}_{i i}(s, t):=\partial Q_{i i}(s, t) / \partial t>0$,

(ii) for all $s \in \mathbf{R}_{+} \exists s_{1}: \dot{\mathbf{Q}}\left(s, s_{1}\right):=\left.(\partial \mathbf{Q}(s, t) / \partial t)\right|_{t=s_{1}}>0$ is a mono-unireducible matrix.

As it is well known, one defines the $n$-fold convolution of $\mathbf{Q}(s, t)$ by itself as:

$$
Q_{i j}^{(n)}(s, t)= \begin{cases}Q_{i j}(s, t), & \text { if } n=1, \\ \sum_{k \in I} \int_{s}^{t} \dot{Q}_{i k}(s, \theta) Q_{k j}^{(n-1)}(\theta, t) d \theta, & \text { if } n \geq 2\end{cases}
$$


Lemma 3.4. Let $Z(t)$ be a NHCTSMP with a continuously differentiable and mono-unireducible kernel $\mathbf{Q}(s, t)$. Then, for all $s \in \mathbf{R}_{+} \exists t \in \mathbf{R}_{+}$such that

$$
\mathbf{Q}^{(n)}(s, t)=\left[\begin{array}{cc}
\mathbf{Q}_{U, U}^{(n)}(s, t) & \mathbf{Q}_{U, D}^{(n)}(s, t) \\
\mathbf{0}^{T} & 1
\end{array}\right]
$$

where $\mathbf{Q}_{U, U}^{(n)}(s, t)>\mathbf{0}, \mathbf{Q}_{U, D}^{(n)}(s, t)>0$.

Proof. Given $s \in \mathbf{R}_{+}$, since $Z(t)$ is mono-unireducible, $\exists s_{1}>s: \mathbf{Q}\left(s, s_{1}\right)$ is a mono-unireducible matrix. Moreover, for all $i, j \in I$ such that $\dot{Q}_{i j}\left(s, s_{1}\right)>0$, because $\dot{Q}_{i j}(s, \cdot)$ is continuous, $\exists I_{i j}\left(s_{1}\right) \subset \mathbf{R}_{+}$: for all $\theta_{1} \in I_{i j}\left(s_{1}\right), \dot{Q}_{i j}\left(s, \theta_{1}\right)>0$. By setting $I\left(s_{1}\right)=\bigcap_{i, j} I_{i j}\left(s_{1}\right)$ we have

$$
\mathbf{Q}^{(n)}(s, t)=\int_{s}^{t} \dot{\mathbf{Q}}\left(s, \theta_{1}\right) * \mathbf{Q}^{(n-1)}\left(\theta_{1}, t\right) d \theta_{1} \geq \int_{I\left(s_{1}\right)} \dot{\mathbf{Q}}\left(s, \theta_{1}\right) * \mathbf{Q}^{(n-1)}\left(\theta_{1}, t\right) d \theta_{1}
$$

and by iteration we get

$$
\mathbf{Q}^{(n)}(s, t) \geq \int_{I\left(s_{1}\right)} \cdots \int_{I\left(s_{n}\right)} \dot{\mathbf{Q}}\left(s, \theta_{1}\right) * \dot{\mathbf{Q}}\left(\theta_{1}, \theta_{2}\right) * \cdots * \dot{\mathbf{Q}}\left(\theta_{n}, t\right) d \theta_{1} d \theta_{2} \cdots d \theta_{n}
$$

The matrices $\mathbf{Q}\left(\theta_{i}, \theta_{i+1}\right)$ are all mono-uniredudible, and then, their product is a monounireducible matrix and for $n>m-1 \mathbf{Q}_{U U}^{(n)}(s, t)$ is a full matrix as a consequence of Theorem 2.1. in [9].

At this point, we can state the following.

Theorem 3.5. Let $Z(t)$ be a NHSMP with continuously differentiable and mono-unireducible kernel $\mathbf{Q}(s, t)$. Then, for all $s \in \mathbf{R}_{+}$

$$
\lim _{t \rightarrow \infty} \Phi(s, t)=\left[\begin{array}{cccc}
0 & \cdots & 0 & 1 \\
\vdots & \ddots & \vdots & \vdots \\
0 & \cdots & 0 & 1
\end{array}\right]
$$

Proof. Let $i \neq m$ then,

$$
\begin{aligned}
\phi_{i m}(s, t)= & P\left[Z(t)=m \mid Z(s)=i, T_{N(s)}=s\right] \\
= & \sum_{k=1}^{m} \int_{0}^{t-\xi-s} P\left[Z(t)=m, Z(t-\xi)=k, B(t-\xi)=l \mid Z(s)=i, T_{N(s)}=s\right] d l \\
= & \sum_{k=1}^{m} \int_{0}^{t-\xi-s} P[Z(t)=m \mid Z(t-\xi)=k, B(t-\xi)=l] \\
& \times P\left[Z(t-\xi)=k, B(t-\xi)=l \mid Z(s)=i, T_{N(s)}=s\right] d l
\end{aligned}
$$




$$
\begin{aligned}
= & \sum_{k=1}^{m} \int_{0}^{t-\xi-s}{ }^{b} \phi_{k m}(t-\xi-l, t-\xi ; t) \phi_{i k}^{b}(s ; t-\xi-l, t-\xi) d l \\
= & \int_{0}^{t-\xi-s}{ }^{b} \phi_{m m}(t-\xi-l, t-\xi ; t) \phi_{i m}^{b}(s ; t-\xi-l, t-\xi) d l \\
& +\sum_{k \neq m} \int_{0}^{t-\xi-s}{ }^{b} \phi_{k m}(t-\xi-l, t-\xi ; t) \phi_{i k}^{b}(s ; t-\xi-l, t-\xi) d l .
\end{aligned}
$$

Then, we get

$$
\phi_{i m}(s, t)-\phi_{i m}(s, t-\xi)=\sum_{k \neq m} \int_{0}^{t-\xi-s^{b}}{ }^{b} \phi_{k m}(t-\xi-l, t-\xi ; t) \phi_{i k}^{b}(s ; t-\xi-l, t-\xi) d l .
$$

In this way, for all $\xi>0, t-\xi>s, \phi_{i m}(s, t) \geq \phi_{i m}(s, t-\xi)$. Moreover $\phi_{i m}(s, t) \leq 1$ which implies, for all $i$ the existence of $\lim _{t \rightarrow \infty} \phi_{i m}(s, t)$.

Let suppose that $\lim _{t \rightarrow \infty} \phi_{i m}=1-\epsilon, 0<\epsilon<1$; then for all $\delta>0 \exists \bar{t}$ : for all $t>\bar{t}$

$$
\sum_{k \neq m} \int_{0}^{t-\xi-s} \phi_{i k}^{b}(s ; t-\xi-l, t-\xi)^{b} \phi_{k m}(t-\xi-l, t-\xi ; t) d l<\delta .
$$

By considering the following inequalities:

$$
\begin{gathered}
\phi_{i k}^{b}(s ; t-\xi-l, t-\xi) \geq Q_{i k}^{(n)}(s, t-\xi-l)\left(1-H_{k}(t-\xi-l, t-\xi)\right), \\
{ }^{b} \phi_{k m}(t-\xi-l, t-\xi ; t) \geq \frac{Q_{k m}^{(2)}(t-\xi-l, t)}{1-H_{k}(t-\xi-l, t-\xi)} \geq \frac{Q_{k k}(t-\xi-l, t-\xi) Q_{k m}(t-\xi, t)}{\left(1-H_{k}(t-\xi-l, t-\xi)\right)},
\end{gathered}
$$

inside (3.8), we get

$$
\sum_{k \neq m} \int_{0}^{t-\xi-s} Q_{i k}^{(n)}(s, t-\xi-l) Q_{k k}(t-\xi-l, t-\xi) Q_{k m}(t-\xi, t) d l<\delta .
$$

Since, by Lemma 3.4, for $n>m, Q_{i k}^{(n)}(s, t-\xi-l)$ is a full matrix, we set $\lambda=$ $\min _{i, k}\left\{Q_{i k}(s, t-\xi-l)\right\}>0$ and $\alpha=\min _{k}\left\{Q_{k k}(t-\xi-l, t-\xi)\right\}>0$. Then, by substitution in (3.10), we have

$$
\sum_{k \neq m} \int_{0}^{t-\xi-s} \lambda \alpha Q_{k m}(t-\xi, t) d l<\delta \Longleftrightarrow(t-\xi-s) \curlywedge \alpha \sum_{k \neq m} Q_{k m}(t-\xi, t)<\delta,
$$


which implies

$$
\sum_{k \neq m} Q_{k m}(t-\xi, t)<\frac{\delta}{(t-\xi-s) \lambda \alpha}
$$

Since $\delta$ is arbitrary, nothing can ensure (3.12) for mono-unireducible semi-Markov process and consequently for all $i, \lim _{t \rightarrow \infty} \phi_{i m}(s, t)=1$.

Corollary 3.6. Let $Z(t)$ be a NHSMP with continuously differentiable and mono-unireducible kernel $\mathbf{Q}(s, t)$. Then for all $s, u, v \in \mathbf{R}_{+}$such that $s-v \leq s \leq s+u$ it results that

$$
\lim _{t \rightarrow \infty}{ }^{b f} \Phi(v, s, u ; t)=\left[\begin{array}{cccc}
0 & \cdots & 0 & 1 \\
\vdots & \ddots & \vdots & \vdots \\
0 & \cdots & 0 & 1
\end{array}\right]
$$

Proof. Because we are interested in the asymptotic behaviour as $t \rightarrow \infty$, it is not a loss of generality to consider that $T_{N(s)+1}<t$.

Let $i \neq m$, then

$$
\begin{aligned}
\lim _{t \rightarrow \infty} P[Z(t)=j \mid Z(s)=i, B(s)=v, F(s)=u] \\
=\sum_{k=1}^{m} \lim _{t \rightarrow \infty} P\left[Z(t)=j, J_{N(s+u)}=k \mid Z(s)=i, B(s)=v, F(s)=u\right] \\
=\sum_{k=1}^{m} \lim _{t \rightarrow \infty} P\left[Z(t)=j \mid J_{N(s+u)}=k, Z(s)=i, B(s)=v, F(s)=u\right] \\
\quad \times P\left[J_{N(s+u)}=k \mid Z(s)=i, B(s)=v, F(s)=u\right] \\
=\sum_{k=1}^{m} \frac{d Q_{i k}(s-v ; s+u)}{1-H_{i}(s-v ; s+u)} \lim _{t \rightarrow \infty} \phi_{k j}(s+u ; t) .
\end{aligned}
$$

Now, from Theorem 3.5 we know that

$$
\lim _{t \rightarrow \infty} \phi_{k j}(s+u, t)= \begin{cases}1 & \forall k \text { if } j=m \\ 0 & \forall k \text { if } j \neq m\end{cases}
$$

consequently if $j \neq m \lim _{t \rightarrow \infty}{ }^{b f} \phi_{i j}(v, s, u ; t)=0$, otherwise for $j=m$, we have that

$$
\lim _{t \rightarrow \infty}^{b f} \phi_{j, m}(v, s, u ; t)=\sum_{k=1}^{m} \frac{d Q_{i k}(s-v ; s+u)}{1-H_{i}(s-v ; s+u)}=1 .
$$

Remark 3.7. From the previous theorem it results that $\left(\phi_{i m}(s, \cdot)\right)$ for all $s \in \mathbf{R}_{+}$and for all $i \in$ $I$ is the distribution function of going into the down state $m$. Moreover $\left({ }^{b f} \phi_{i m}(v, s, u ; \cdot)\right)$, 
for all $v, s, u \in \mathbf{R}_{+}$and for all $i \in I$ is the distribution function of going into the down state $m$ given that at time $s$ the process is in state $i$ with a backward and forward values of $v$ and $u$, respectively.

\section{The Credit Risk Model}

SMP were proposed to describe rating dynamics for the first time by [10] with the purpose of construct efficient rating migration models. Efficient migration models are of interest for pricing rating sensitive derivatives (see $[11,12])$, for the valuation of portfolio of defaulting bonds, for credit risk management and capital allocation; see [13, 14].

Here, we apply our non-homogeneous model to entity ratings history, instrument ratings history, and issue/maturity ratings history, respectively for each standard \& poor's rated entity, for each Standard \& Poor's rated instrument stock or bonds sold by an entity at particular time, and issue/maturity for the Global Issuers and Structured Finance (GI and FS) instruments that Standard \& Poor's has rated, in the GI\&SF sector formerly known as the Corporate Finance since 1922 to 2007, July, 16.

In the Standard \& Poor's case there are 8 different classes of rating expressing the creditworthiness of the rated firm. The ratings are listed to form the following set of states:

$$
I=\{A A A, A A, A, B B B, B B, B, C C C, D\} .
$$

The creditworthiness is highest for the rating $A A A$, assigned to firm extremely reliable with regard to financial obligations and decrease towards the rating $D$ which expresses the occurrence of payment default on some financial obligation. A table showing the financial meaning of the Standard \& Poor's rating categories is reported in [15]. As a matter of example, the rating $A$ is assigned to firm susceptible to economic conditions having still good credit quality.

The state space is partitioned in up and down states as follows:

$$
U=\{A A A, A A, A, B B B, B B, B, C C C\}, \quad D=\{D\} .
$$

To dispose of a semi-Markov model it is necessary to construct the embedded Markov chain $\mathbf{P}(s)$ and to find the d.f. of waiting times $\mathbf{G}(s, t)$. The embedded Markov chain, constructed by rating agency Standard \& Poor's (S\&P) real data, was given in [16] and is reported in Tables 1, 2, and 3 for different years.

Remark 4.1. We would outline that the Markov matrices that are given yearly in the S\&P publications are usually diagonal dominant. Instead, the matrices that are presented in this paper do not have this property. The reason is that in a semi-Markov environment the transitions are taken into account only if the rating agency assesses a new rating. On the contrary, in the evaluation of the S\&P transition Markov chain, if in a year there was no new rating evaluation for a firm, it is supposed that the firm is yet in the same state, and the rating agency in the construction of the transition matrix takes into account that there is a "virtual" transition in the same state. This implies that the number of virtual transitions is very high and the Markov chain becomes diagonally dominant. We think that this is another reason of the best behaviour of the semi-Markov environment with respect to the Markov one, because only the real rating transitions are considered. 
Table 1: Embedded Markov chain for $s=1$.

\begin{tabular}{lcccccccc}
\hline & $A A A$ & $A A$ & $A$ & $B B B$ & $B B$ & $B$ & $C C C$ & $D$ \\
\hline$A A A$ & 0.623 & 0.319 & 0.043 & 0.015 & 0.000 & 0.000 & 0.000 & 0.000 \\
$A A$ & 0.090 & 0.650 & 0.250 & 0.000 & 0.010 & 0.000 & 0.000 & 0.000 \\
$A$ & 0.017 & 0.123 & 0.714 & 0.132 & 0.014 & 0.000 & 0.000 & 0.000 \\
$B B B$ & 0.000 & 0.011 & 0.365 & 0.581 & 0.043 & 0.000 & 0.000 & 0.000 \\
$B B$ & 0.000 & 0.000 & 0.000 & 0.500 & 0.249 & 0.187 & 0.064 & 0.000 \\
$B$ & 0.000 & 0.199 & 0.000 & 0.200 & 0.000 & 0.000 & 0.601 & 0.000 \\
$C C C$ & 0.000 & 0.000 & 0.000 & 0.000 & 0.143 & 0.286 & 0.143 & 0.428 \\
$D$ & 0.000 & 0.000 & 0.000 & 0.000 & 0.000 & 0.000 & 0.000 & 1.000 \\
\hline
\end{tabular}

Table 2: Embedded Markov chain for year $s=11$.

\begin{tabular}{lcccccccr}
\hline & $A A A$ & $A A$ & $A$ & $B B B$ & $B B$ & $B$ & $C C C$ & $D$ \\
\hline$A A A$ & 0.612 & 0.274 & 0.064 & 0.020 & 0.007 & 0.017 & 0.004 & 0.002 \\
$A A$ & 0.104 & 0.623 & 0.225 & 0.024 & 0.004 & 0.006 & 0.012 & 0.002 \\
$A$ & 0.026 & 0.138 & 0.625 & 0.137 & 0.041 & 0.031 & 0.001 & 0.001 \\
$B B B$ & 0.029 & 0.051 & 0.195 & 0.540 & 0.131 & 0.039 & 0.011 & 0.003 \\
$B B$ & 0.037 & 0.006 & 0.025 & 0.187 & 0.481 & 0.218 & 0.043 & 0.003 \\
$B$ & 0.011 & 0.021 & 0.015 & 0.047 & 0.147 & 0.473 & 0.242 & 0.044 \\
$C C C$ & 0.000 & 0.000 & 0.000 & 0.000 & 0.028 & 0.113 & 0.634 & 0.225 \\
$D$ & 0.000 & 0.000 & 0.000 & 0.000 & 0.000 & 0.000 & 0.000 & 1.000 \\
\hline
\end{tabular}

Table 3: Embedded Markov chain for $s=21$.

\begin{tabular}{lcccccccc}
\hline & $A A A$ & $A A$ & $A$ & $B B B$ & $B B$ & $B$ & $C C C$ & $D$ \\
\hline$A A A$ & 0.747 & 0.139 & 0.074 & 0.034 & 0.004 & 0.002 & 0.000 & 0.000 \\
$A A$ & 0.052 & 0.573 & 0.309 & 0.036 & 0.026 & 0.002 & 0.001 & 0.001 \\
$A$ & 0.024 & 0.180 & 0.539 & 0.240 & 0.011 & 0.003 & 0.002 & 0.001 \\
$B B B$ & 0.007 & 0.039 & 0.228 & 0.461 & 0.229 & 0.033 & 0.001 & 0.002 \\
$B B$ & 0.005 & 0.024 & 0.048 & 0.426 & 0.307 & 0.175 & 0.012 & 0.003 \\
$B$ & 0.002 & 0.014 & 0.016 & 0.096 & 0.371 & 0.341 & 0.150 & 0.010 \\
$C C C$ & 0.000 & 0.010 & 0.015 & 0.070 & 0.120 & 0.525 & 0.165 & 0.095 \\
$D$ & 0.000 & 0.000 & 0.000 & 0.000 & 0.000 & 0.000 & 0.000 & 1.000 \\
\hline
\end{tabular}

By using the empirical estimator of cumulative distribution functions, we estimated the distributions $G_{i, j}(s, t)$. In Figure 2, we report only few cases.

By using relation (2.5), we recover the semi-Markov kernel, and the application of the theoretical arguments of previous sections allow us to compute the transition probabilities. Following the approach in $[2,10]$ we denote by

$$
{ }^{b} R_{i}(l, s ; t):=P[Z(t) \in U \mid Z(s)=i, B(s)=s-l]=\sum_{j \in U}^{b} \phi_{i j}(l, s ; t),
$$

the reliability function with initial backward. It expresses the probability that the firm has never defaulted up to time $t$ given that at time $s$ it was in rating class $i \in E$, where it entered with last transition at time $s-l$.

It is simple to realize that $1-^{b} R_{i}(l, s ; t)$ is the probability to be defaulted at time $t$. 


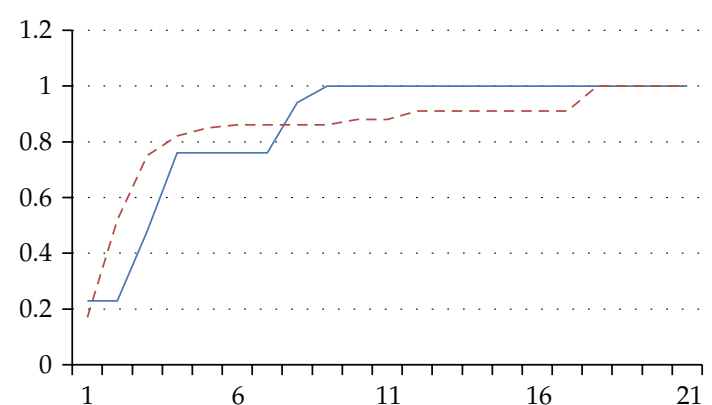

(a)

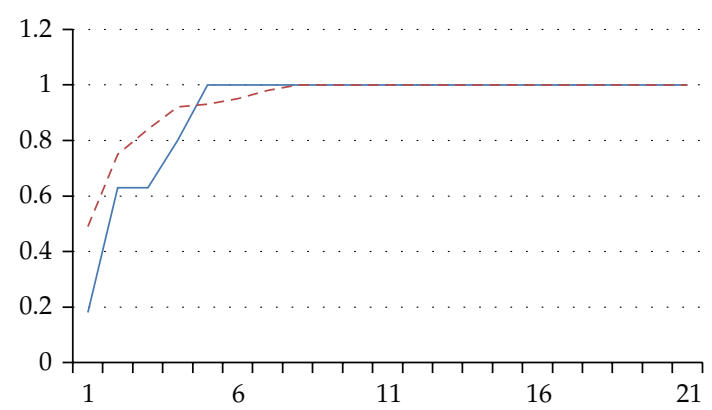

(c)

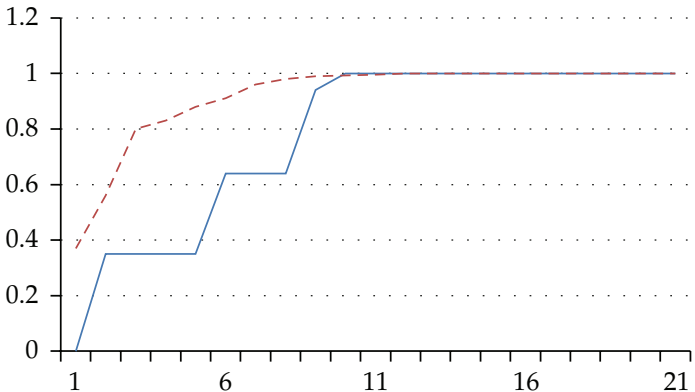

(b)

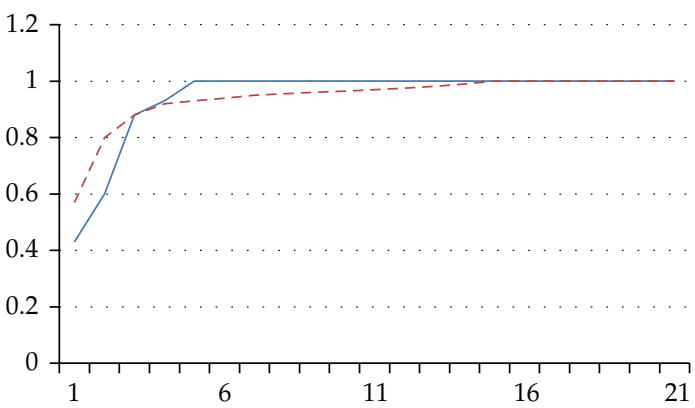

(d)

Figure 2: Conditional waiting time d.f. $G_{A A A, A A A}(s, s+k)$ (dotted lines) and $G_{A, A A A}(s, s+k)$ (continuous lines) for $s=1(\mathrm{a}), s=3$ (b), $s=6(\mathrm{c})$, and $s=9$ (d).

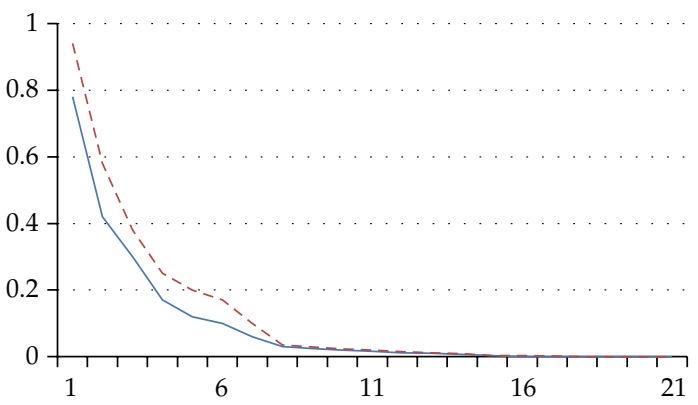

(a)

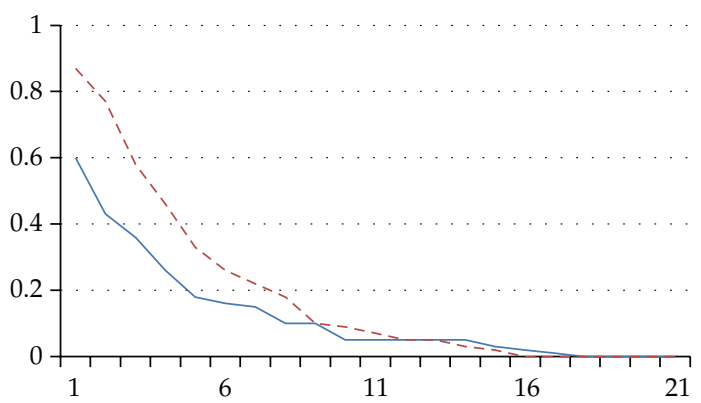

(c)

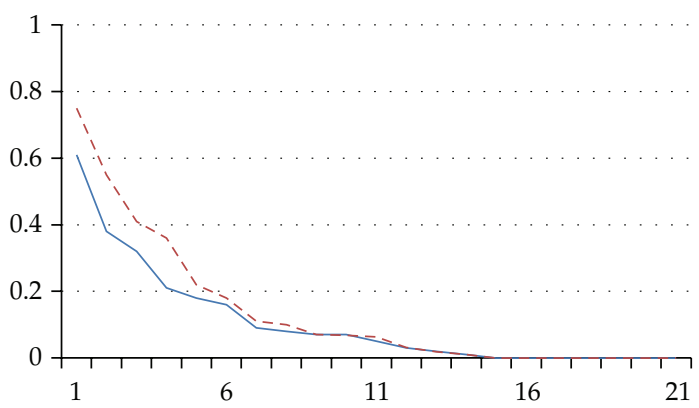

(b)

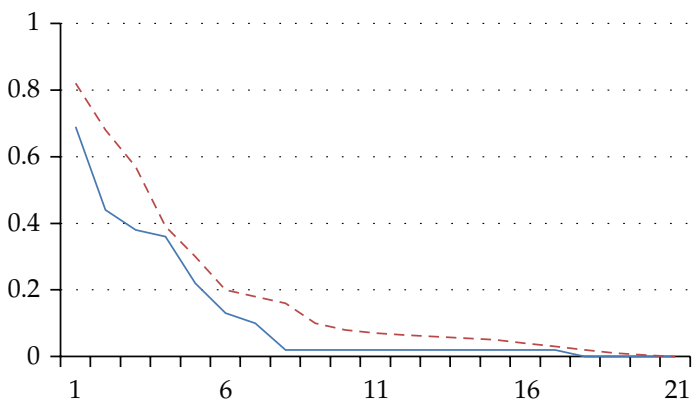

(d)

Figure 3: Reliability function ${ }^{b} R_{A}(l, s ; s+k)$ for $s=4$ (dotted lines) and $s=8$ (continuous lines) for $l=1$ (a), $l=2(\mathrm{~b})$, and $l=3(\mathrm{~b}), l=4(\mathrm{~d})$. 
In Figure 3, we show the reliability function for rating class $i=A$ for different values of $l, s$, and $t$. As it is possible to see, the reliabilities have a different behaviour depending on the values of $s$. This is due to the nonhomogeneity of the rating process. The reliability exhibits also variability as a function of the backward process, then as a result, our model assigns different survival probabilities to firms having the same rating class but with different age in this state.

\section{References}

[1] J. Janssen and R. Manca, Semi-Markov Risk Models for Finance, Insurance and Reliability, Springer, New York, NY, USA, 2007.

[2] G. D'Amico, J. Janssen, and R. Manca, "Initial and final backward and forward discrete time nonhomogeneous semi-Markov credit risk models," Methodology and Computing in Applied Probability, vol. 12, no. 2, pp. 215-225, 2010.

[3] G. D'Amico, J. Janssen, and R. Manca, "Semi-Markov reliability models with recurrence times and credit rating applications," Journal of Applied Mathematics and Decision Sciences, vol. 2009, Article ID 625712, 17 pages, 2009.

[4] J. Yackel, "Limit theorems for semi-Markov processes," Transactions of the American Mathematical Society, vol. 123, pp. 402-424, 1966.

[5] E. Çinlar, "Markov renewal theory," Advances in Applied Probability, vol. 1, pp. 123-187, 1969.

[6] N. Limnios and G. Oprişan, Semi-Markov Processes and Reliability Modelling, Birkhäuser Boston Inc., Boston, Mass, USA, 2001.

[7] V. Korolyuk and A. Swishchuk, Semi-Markov Random Evolutions, Kluwer Academic Publishers, Dodrecht, The Netherlands, 1995.

[8] G. D'Amico, J. Janssen, and R. Manca, “Duration dependent semi-Markov models," Applied Mathematical Sciences, vol. 5, no. 41-44, pp. 2097-2108, 2011.

[9] G. D'Amico, J. Janssen, and R. Manca, "The dynamic behaviour of non-homogeneous singleunireducible Markov and semi- Markov chains," in Networks: Topology and Dynamic Lectures Notes in Economic and Mathematical Systems, pp. 195-211, Springer, New York, NY, USA, 2009.

[10] G. D'Amico, J. Janssen, and R. Manca, “Homogeneous semi-Markov reliability models for credit risk management," Decisions in Economics and Finance, vol. 28, no. 2, pp. 79-93, 2005.

[11] A. Vasileiou and P.-C. G. Vassiliou, "An inhomogeneous semi-Markov model for the term structure of credit risk spreads," Advances in Applied Probability, vol. 38, no. 1, pp. 171-198, 2006.

[12] G. D'Amico, J. Janssen, and R. Manca, "Valuing credit default swap in a non-homogeneous semiMarkovian rating based model," Computational Economics, vol. 29, no. 2, pp. 119-138, 2007.

[13] D. Lando, Credit Risk Modeling, Princeton University Press, Princeton, NJ, USA, 2004.

[14] S. Trueck and S. T. Rachev, Rating Based Modeling of Credit Risk, Academic Press, New York, NY, USA, 2009.

[15] C. Bluhm, L. Overbeck, and C. Wagner, An introduction to Credit Risk Modeling, CRC Financial Mathematics Series, Chapman \& Hall, Boca Raton, Fla, USA, 2002.

[16] G. D'Amico, G. Di Biase, J. Janssen, and R. Manca, "Homogeneous and non-homogeneous semiMarkov backward credit risk migration models," in Financial Hedging, chapter 1, Nova Science Publishers, New York, NY, USA, 2009. 


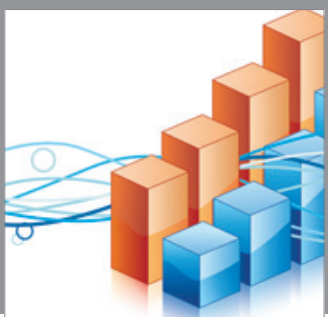

Advances in

Operations Research

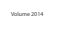

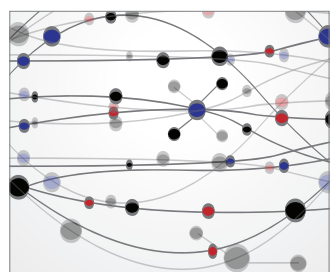

\section{The Scientific} World Journal
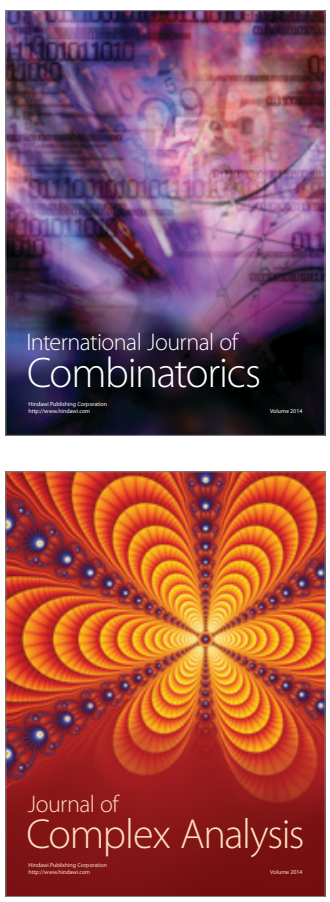

International Journal of

Mathematics and

Mathematical

Sciences
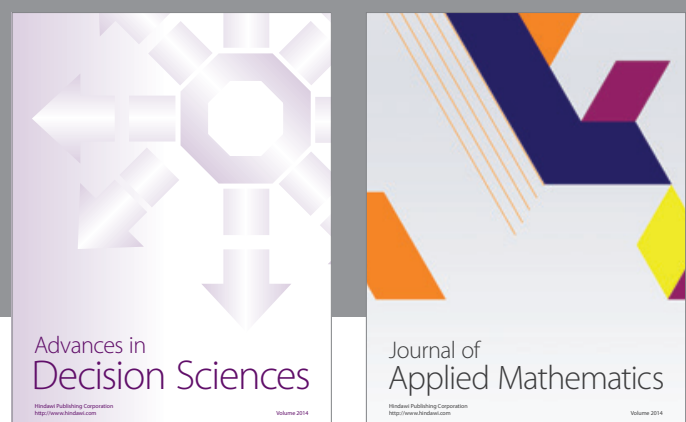

Journal of

Applied Mathematics
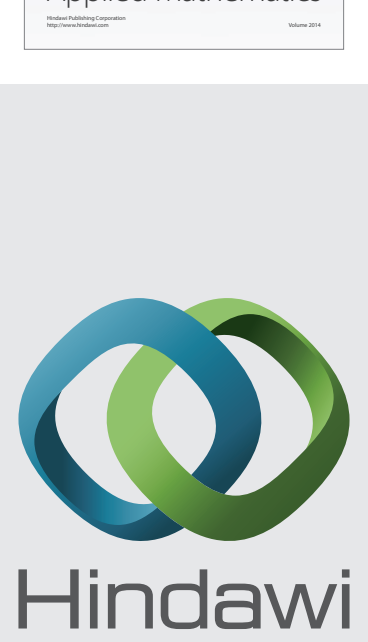

Submit your manuscripts at http://www.hindawi.com
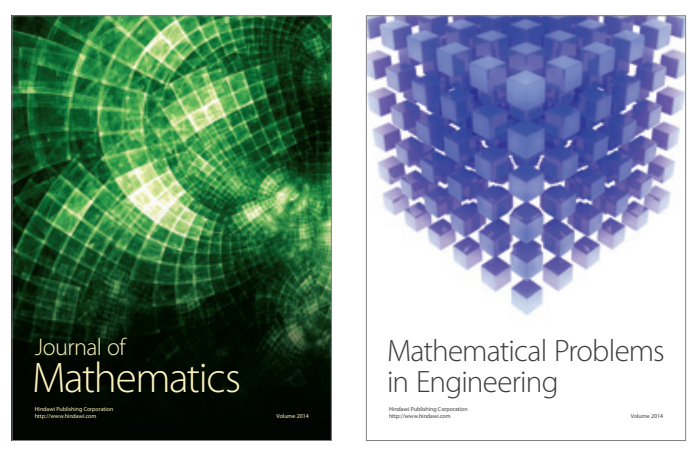

Mathematical Problems in Engineering
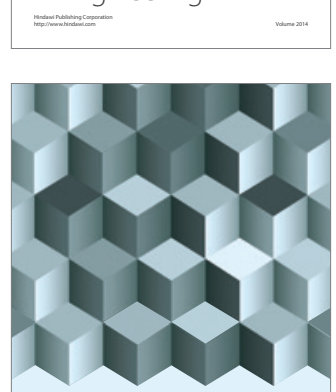

Journal of

Function Spaces
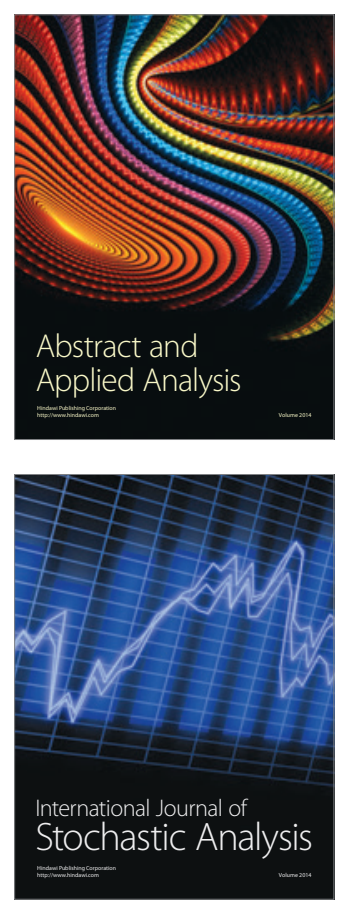

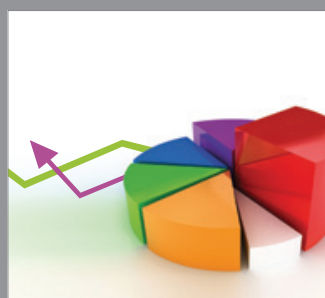

ournal of

Probability and Statistics

Promensencen
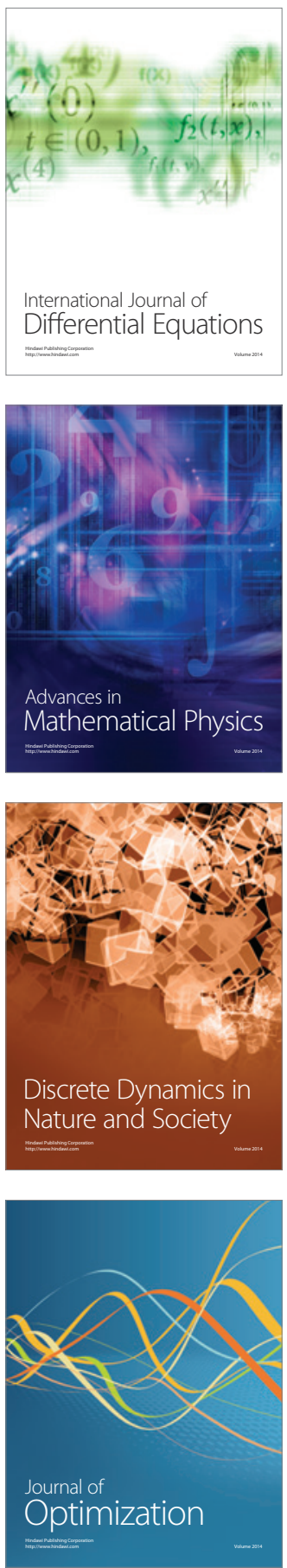